Vie et mort des revues en période coloniale et postcoloniale

\title{
Genèse de Forge et Terrasses
}

Deux revues littéraires dans l'Algérie coloniale

\section{Guy Dugas}

\section{(2) OpenEdition}

\section{Journals}

Édition électronique

URL : http://journals.openedition.org/coma/938

DOI : $10.4000 /$ coma.938

ISSN : 2275-1742

Éditeur

Institut des textes \& manuscrits modernes (ITEM)

Référence électronique

Guy Dugas, "Genèse de Forge et Terrasses », Continents manuscrits [En ligne], 9 | 2017, mis en ligne le 11 octobre 2017, consulté le 24 avril 2019. URL : http://journals.openedition.org/coma/938 ; DOI : 10.4000/coma.938

Ce document a été généré automatiquement le 24 avril 2019.

\section{cc)}

Continents manuscrits - Génétique des textes littéraires - Afrique, Caraîbe, dispora est mis à disposition selon les termes de la licence Creative Commons Attribution - Pas d'Utilisation Commerciale - Pas de Modification 4.0 International. 


\title{
Genèse de Forge et Terrasses
}

\author{
Deux revues littéraires dans l'Algérie coloniale
}

\section{Guy Dugas}

\section{À la mémoire d'Hamid Nacer Khodja}

Les deux revues considérées - Forge (déc. 1946-nov. 1947) et Terrasses (un seul numéro paru en 1953) - ont été fondées à Alger, à l'initiative de deux Européens d'Algérie, l'un et l'autre de retour au pays après plusieurs années passées en métropole. Emmanuel Roblès (1914-1995), écrivain déjà reconnu, obtiendra le prix Femina quelque mois plus tard pour Les Hauteurs de la ville. Jean Sénac (1926-1973), jeune poète qui se cherche encore, vient de passer plusieurs mois en France grâce à une bourse de création.

\section{Le contexte de l'après-guerre}

Dans l'entre-deux-guerres, la puissante Association des écrivains algériens (AEA, fondée

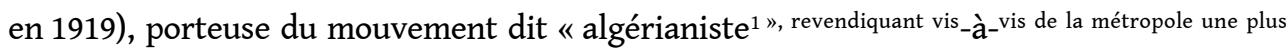
grande autonomie politique et culturelle tout en niant toute culture indigène et en maintenant un très fort clivage entre langue arabe et langue française, règne sur la vie littéraire et culturelle de la colonie; avec un large soutien financier du Gouvernement général de l'Algérie et sous le parrainage de l'académicien Louis Bertrand, elle publie depuis 1924 la revue mensuelle Afrique et garde la haute main sur le très convoité Grand Prix littéraire d'Algérie décerné à partir de 1921 et jusqu'en 1961, non sans quelques interruptions.

Dans l'immédiat après-guerre, cette activité s'est considérablement essouflée: le Gouvernement général n'est plus en mesure de financer aussi généreusement l'Association des écrivains algériens; le Grand Prix littéraire d'Algérie a été très irrégulièrement décerné durant les années de guerre. Cette activité est concurrencée par les entreprises novatrices du groupe de jeunes artistes groupés autour de l'éditeur Charlot (théâtre du Travail et revue Rivages), 
Fig. $1 \mathrm{a}$ et $1 \mathrm{~b}$ : Prière d'insérer de Rivages, signé Albert Camus.
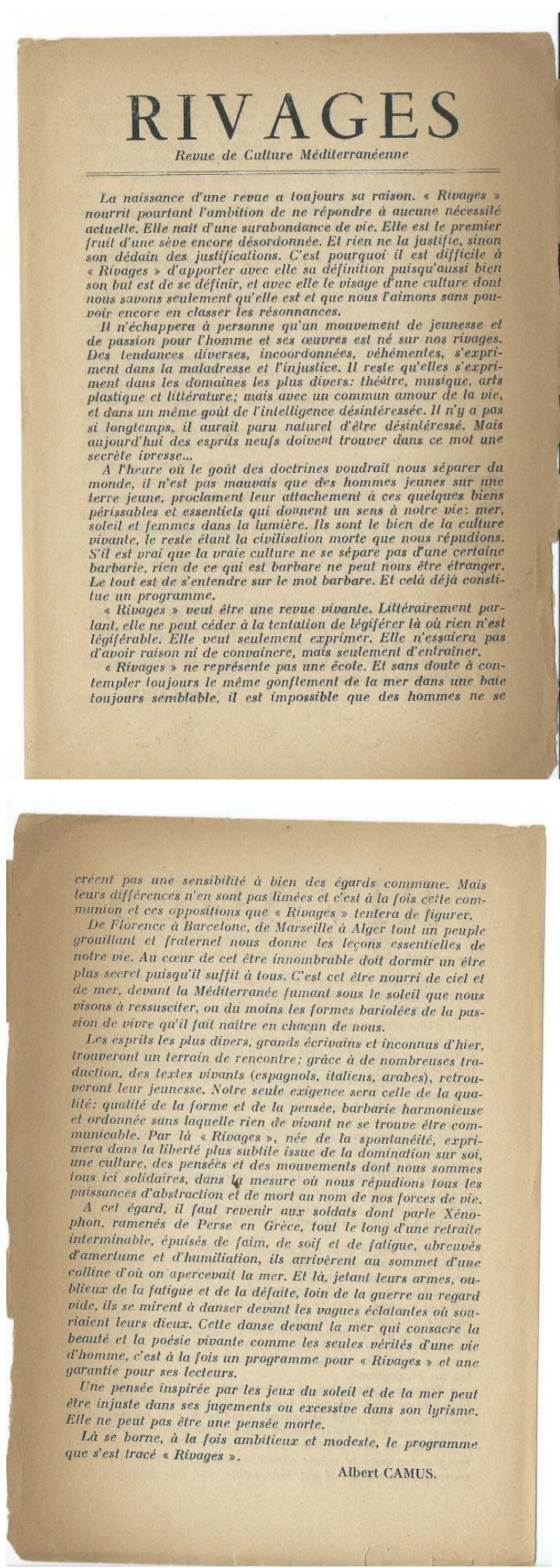

Fonds Patrimoine méditerranéen, BIU Montpellier, section Lettres.

puis par les revues nées de la guerre (Fontaine, L'Arche), qui bénéficient d'un meilleur approvisionnement en papier, Afrique, devenue bimestrielle dans l'été 1942, perd de sa régularité et de son volume. 
Par ailleurs, l'influence politique et culturelle croissante d'États étrangers et certains événements marquants, comme les soulèvements de Sétif et Guelma en mai 1945, contraignent les autorités, lorsqu'elles prennent conscience du fossé qui s'est creusé entre les communautés, à tenter quelques expériences de partage, comme les rencontres de Sidi Madani, initiées par le service des mouvements de jeunesse et d'éducation populaire, rattaché au Gouvernement général.

C'est ce tournant politique et culturel, simultanément porteur de promesses de renouvellement et révélateur de leurs limites dans le contexte politique du moment, que nous voudrions saisir, de manière comparative, à travers la genèse de deux revues essentiellement littéraires, Forge (cinq numéros, dont un double, en 1946-1947) et Terrasses (un numéro unique en juin 1953).

\section{Les hommes...}

Emmanuel Roblès a 32 ans au moment où il crée sa revue. Très tôt, il a entrepris de se démarquer de l'emprise des algérianistes, dont il se montre depuis ses débuts l'un des contempteurs les plus virulents ${ }^{2 .}$ Dans la proximité d'Edmond Charlot et d'Albert Camus, il a participé avant-guerre à la malheureuse mais enrichissante aventure de Rivages ${ }^{3}$. Durant les années suivantes, il voyage beaucoup, en tant que correspondant de guerre, sans que cela ne l'interrompe dans ses projets d'écriture : la publication des Hauteurs de la ville, la création de Montserrat, à Alger et Paris simultanément, vont faire de lui, avec Camus, l'un des écrivains les plus en vue de la colonie, notoriété dont il saura se servir pour développer son projet de revue.

Dès sa démobilisation et son retour en Algérie fin 1945, conscient du gouffre qui est en train de se creuser entre les communautés, il conçoit le projet de fonder des «cahiers littéraires » ambitionnant de rapprocher enfin « sur cette terre du Maghreb, [...] le plus généreux de la pensée islamique d'hier et d'aujourd'hui du plus noble de la pensée française d'hier et d'aujourd'hui ${ }^{4}$ ».

Pour cela il s'associe à deux personnalités connues pour un engagement sans compromission : El Boudali Safir, musicologue et pionnier des émissions en langue arabe sur Radio-Alger, et Louis Julia, journaliste communiste militant et collaborateur d'Alger républicain. À partir de ce premier cercle, élargi à des collaborateurs musulmans de tout premier plan, comme Saâdedine Bencheneb ou Mohammed Zerrouki, ainsi qu'à des amis disposant de leur propre réseau - Émile Dermenghem en Algérie; Ahmed Sefrioui au Maroc; Georges-Albert Astre, qui a créé et animé, en Tunisie, pendant la guerre, le mensuel L'Afrique littéraire, artistique et scientifique où Roblès a publié -, la revue va pouvoir rayonner dans tout le Maghreb, et au-delà dans l'ensemble du monde méditerranéen et arabe, ce que n'avait pu faire Rivages, par manque de durée ou manque d'ambition.

Parmi cet aréopage d'animateurs, Louis Julia (1907-1954) mérite une mention particulière : enseignant dans un collège d'Alger après avoir été le professeur de Roblès au collège Ardaillon d'Oran, militant communiste, c'est par son intermédiaire qu'est obtenu d'Alger républicain, où il tient régulièrement une chronique souvent imprécatoire ${ }^{5}$, le (mauvais) papier sur lequel est imprimé Forge. C'est grâce à lui également que la revue est imprimée sur les presses du journal, rue Koechlin, dont il est un habitué ; sans doute aussi grâce à lui que la revue bénéficie de placards publicitaires sur Alger républicain, «le grand quotidien du matin au service de la Vérité » $\left(\mathrm{n}^{\circ} 1\right)$ ou sa structure éditoriale $\left(\mathrm{n}^{\circ \mathrm{s}} 1\right.$, $3,4)$ à laquelle la revue est adossée... Si bien que la disparition de Forge sera largement 
causée par un conflit avec Louis Julia et Alger républicain, qui privera ses camarades de recettes publicitaires indispensables et de toute possibilité d'impression ${ }^{6}$ !

Beaucoup moins émancipé que son aîné des vieux sentiments algérianistes, Jean Sénac n'a que 27 ans au moment où il crée Terrasses et sa notoriété ne dépasse guère les frontières de la colonie. Il n'a rien d'un gestionnaire, mais il a acquis dans la direction de revues une certaine expérience et il a pu tirer les leçons des échecs, mais aussi de certaines réussites, des deux qu'il a précédemment fondées : avec M... (un numéro en mars 1949), entreprise très artisanale et confidentielle, due à l'initiative de trois jeunes poètes isolés et impécunieux (Jacques Lévy, Philippe Louit et Jean Sénac), puis Soleil (sept numéros, dont un double, de 1950 à 1952), beaucoup plus ambitieuse dans sa présentation (couverture bichrome et illustrations in-textes) et dans son projet («marquer au milieu des divergences et des inquiétudes la fraternité et l'espoir des hommes $\left.{ }^{7} »\right)$, il s'est constitué un réseau de collaborateurs potentiels et dispose, en Algérie et en France au moins, de quelques bonnes adresses.

Parmi les initiateurs de ces trois publications, d'une moyenne d'âge bien moindre que celle des collaborateurs de Forge, on note la présence de plusieurs animateurs de RadioAlger, station qui se comportera comme une chambre d'écho de ces revues, en organisant des lancements, des émissions spéciales et des expositions, principalement sur la série de Soleil.

\section{Présentation et logistique}

Les cahiers de Forge, au format $14 \times 22,5 \mathrm{~cm}$, sont imprimés sur un papier de médiocre qualité, terne et cassant, sous couverture bicolore d'un grammage insuffisant qui présente le sommaire détaillé de chaque numéro - tout cela conduisant avec le temps à des fascicules assez ressemblants aux ouvrages publiés par Charlot pendant la guerre. La quatrième de couverture est muette, à l'exception de l'indication du prix au numéro : 40 francs. 
fig. 2 : Couverture du n ${ }^{\circ} 1$ de Forge.

(Cette couverture ne connaîtra aucune évolution du $1^{\mathrm{er}}$ au dernier numéro.)

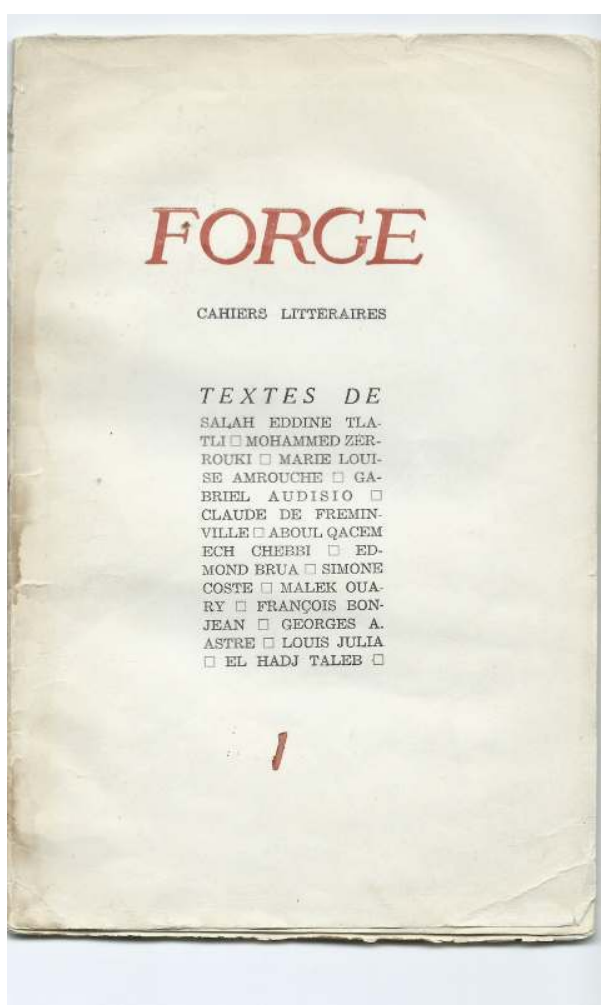

Fonds Patrimoine méditerranéen, BIU Montpellier, section Lettres.

L'adresse de la revue, rappelée dans chaque numéro, est au 9, rue Koechlin, siège d'Alger $r$ épublicain. Il n'y a pas de nom de gérant ; trois correspondants à Paris, Rabat et Tunis sont désignés. Dans les deux premiers numéros, une page vaut bulletin de souscription pour six cahiers - cet engagement expliquant probablement l'ultime numéro double ( $\left.{ }^{\circ} 5-6\right)$. Chaque fascicule s'achève sur un cahier d'annonces publicitaires, dont le plus grand nombre renvoie aux métiers du livre : nombreuses publicités des éditions Charlot ou En Nadha, des éditions parisiennes du club Maintenant, ou encore d'imprimeurs ou de libraires locaux; mais également des placards pour des commerces extrêmement divers : huilerie, compagnie d'assurance, miroiterie d'art, et même pour une marque de cigarettes désignée comme « la cigarette des sportifs »!

D'un format légèrement plus grand $(16,5 \times 24 \mathrm{~cm})$, Terrasses bénéficie d'un papier de bien meilleure qualité - les temps de pénurie sont visiblement achevés. La revue, dont le comité de rédaction se compose de onze personnes (contre trois seulement pour Forge), se domicilie au 41 de la rue Mogador, siège de l'imprimerie Heintz, qui la réalise ; Jean Sénac en est le directeur-gérant. Très sobre, la couverture, imprimée en lettres noires sur fond jaune, annonce une "nouvelle revue algérienne ${ }^{8}$ ", et déroule le sommaire complet, à la manière des couvertures de Forge. 
fig. 3 : Couverture de l'unique numéro de Terrasses.

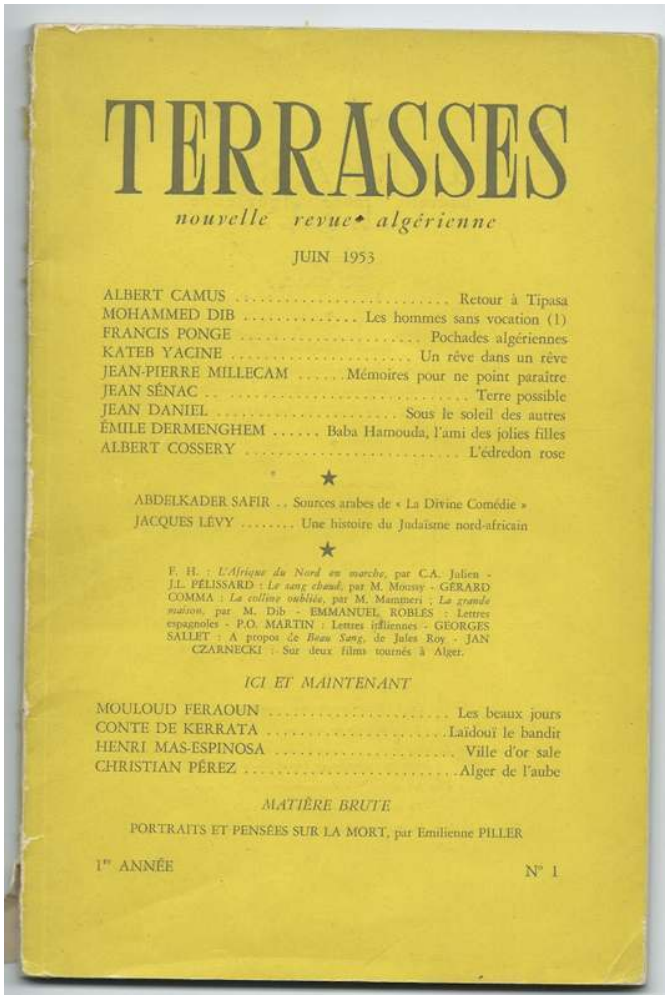

Fonds Patrimoine méditerranéen, BIU Montpellier, section Lettres.

En revanche, contrairement à celle de Forge, la quatrième de couverture n'est pas muette, puisqu'elle présente, outre le prix au numéro ( 250 francs), les cinq premiers titres de la collection «Méditerranée », dirigée depuis peu aux éditions du Seuil par... Emmanuel Roblès! 


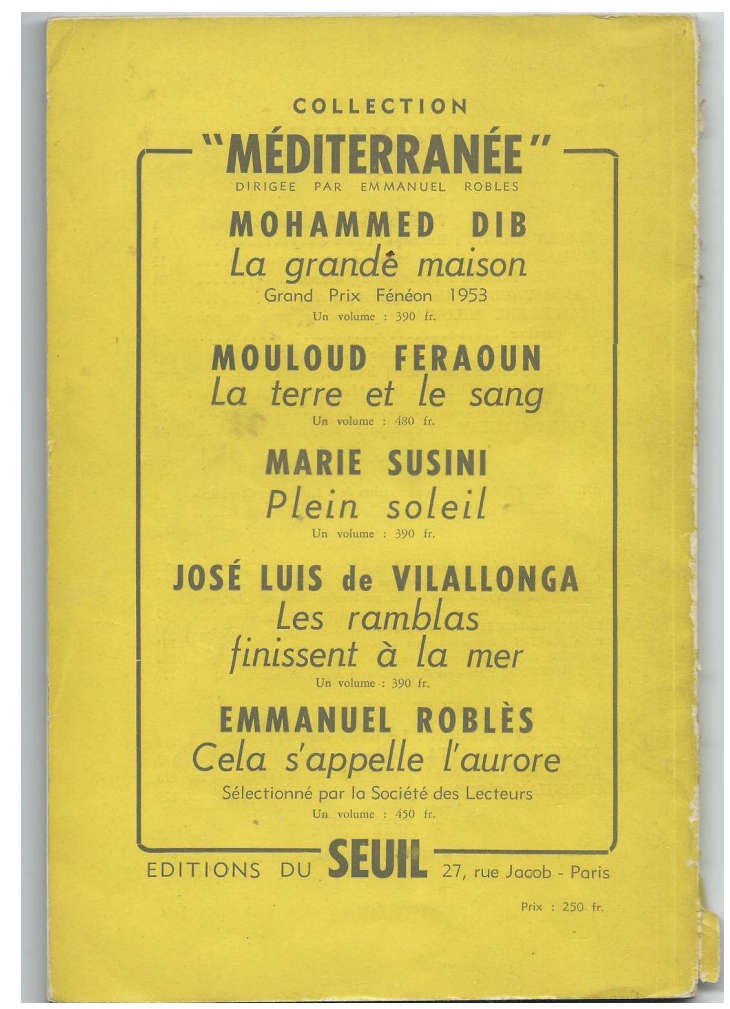

Fonds Patrimoine méditerranéen, BIU Montpellier, section Lettres.

Autre différence notable avec Forge: Terrasses ne contient pas le moindre placard publicitaire, en dehors de la quatrième de couverture. Non que les promoteurs de la revue ne l'aient souhaité', mais les temps ont changé : nous sommes à la veille de l'insurrection nationale et une méfiance générale s'est installée. Par ailleurs, les éditions Charlot, principal support publicitaire de revues comme Forge ou L'Arche, ont disparu.

C'est donc ailleurs que devront être trouvés les moyens de financement. Pas de soucis pour le premier numéro, « entièrement payé avec l'argent que l'oncle m'a laissé10 ». Mais, avant même la sortie du $n^{\circ} 1$, la suite est déjà problématique. Semaine après semaine, Sénac doit harceler l'administration coloniale pour une subvention, les milieux littéraires maghrébins pour des abonnements, ses propres partenaires pour qu'ils trouvent de la publicité. À Albert Memmi, il écrit : «Grâce à la publicité, je crois que nous tiendrons le coup. Je prévois d'autre part la création d'une Société civile pour l'exploitation de la revue $^{11}$.»

\section{Projet et ambitions}

\section{Forge}

Dans une adresse non signée «À nos lecteurs" publiée en fin $d u n^{\circ} 1$, puis, plus logiquement, en tête des suivants, l'équipe de Forge explicite son projet : au lendemain des graves événements du 8 mai 1945, au moment même des rencontres de Sidi Madani, auxquelles Sénac et Roblès ont activement participé et au cours desquelles ils ont noué d'utiles relations ${ }^{12}$, il s'agit de «forger de toniques amitiés ${ }^{13}$ » au sein de la société algérienne et bien au-delà des frontières. 
On voit vers quel champ sémantique nous entrainent les termes «forge » et "forger ", faisant écho à la sobriété de la couverture de la revue. Ils empruntent à l'univers des petits travailleurs, des artisans, si présents dans l'œuvre de Roblès, et font référence à un travail, ardu et collectif, mais qui met le fer au feu et agit sur la matière. C'est dire qu'il s'agit bien d'une entreprise volontariste et de résistance d'une poignée d'« hommes de bonne volonté ${ }^{14} »$ restés optimistes dans leurs espoirs de dépassement de tous les clivages ethniques, linguistiques et idéologiques des sociétés coloniales.

De manière plus concrète, plus ancrée en son temps que Rivages - dont le paratexte n'évoque pour tout programme, afin de conjurer «les puissances d'abstractions et de mort ", qu'une "danse devant la mer" qui ne s'aventure guère plus loin que "de Florence à Barcelone [et] de Marseille à Alger "-, l'adresse de Forge à ses lecteurs oppose tout du long ce «monde dynamique et mouvant qu'est l'Afrique du Nord» aux « soucis immédiats et cruels des uns et des autres ${ }^{15} "$, avec l'espoir implicite de pouvoir agir pour «la libération des esprits que trop d'ignorances et de préjugés emprisonnent ».

$\mathrm{Au}$ bout du compte, Forge restera la première revue algérienne à avoir publié, en édition bilingue, de grands auteurs maghrébins de langue arabe, comme Mahmoud Taha, Abdul Kacem Chabbi, ou encore Mohammed Al Id Hammou Ali.

fig. 5 : Poème d'Al Id Hammou Ali en édition bilingue (Forge $\mathrm{n}^{\circ} 3$ ).

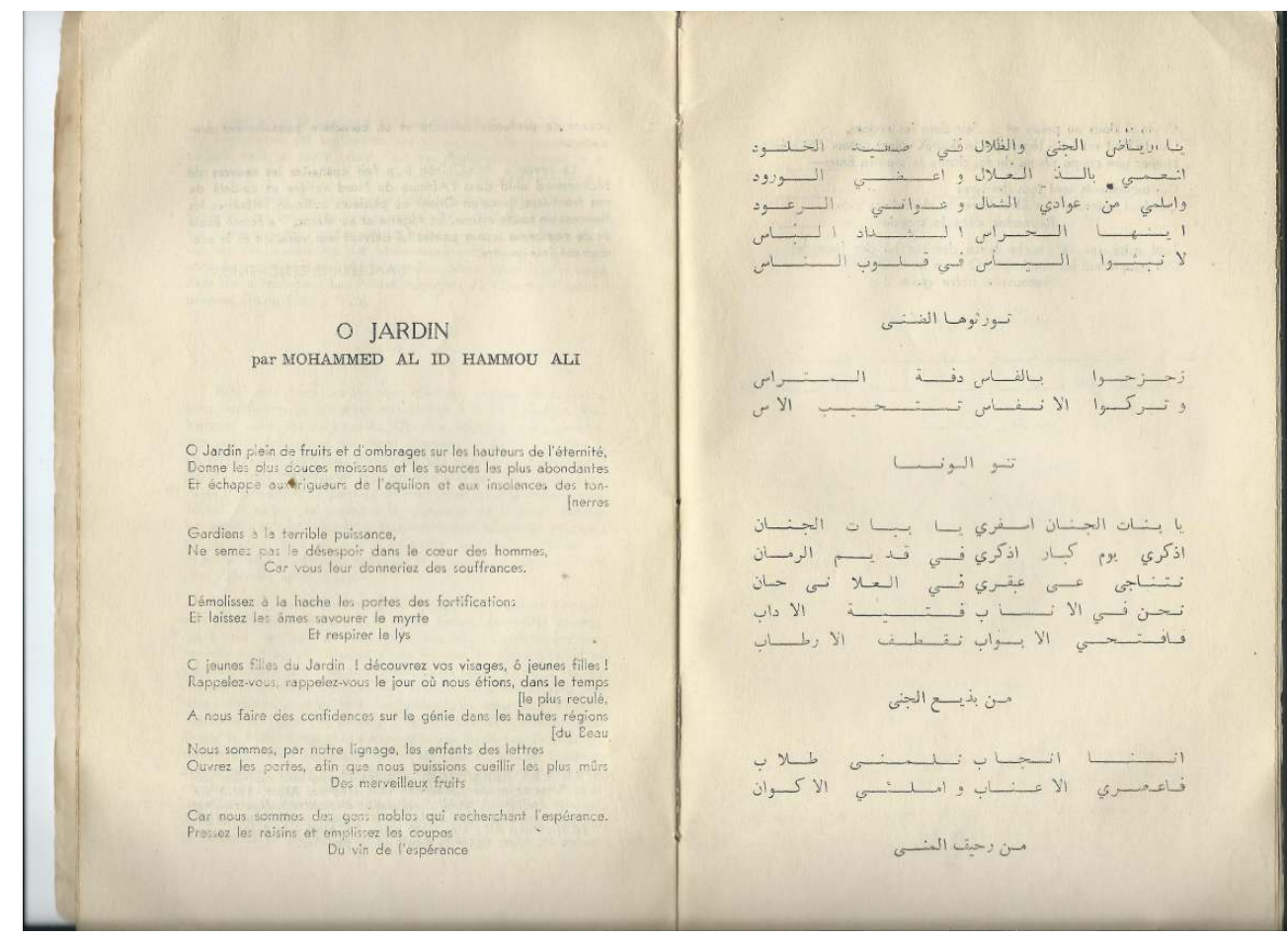

Fonds Patrimoine méditerranéen, BIU Montpellier, section Lettres.

Même si, paradoxalement, la petite anthologie de «La jeune poésie nord-africaine » du ${ }^{\circ}$ 3 ne met à l'honneur que des poètes de langue française, comme si la modernité littéraire ne pouvait passer que par cette langue.

En outre, la position de Roblès au cœur de la vie culturelle franco-algérienne, au centre de la galaxie Charlot alors au summum de son rayonnement ainsi que l'ancrage de la revue au sein de la puissante société de presse et d'édition d'Alger républicain, lui permet 
d'attirer vers Forge de jeunes auteurs encore inconnus comme Mohammed Dib, Kateb Yacine (qui signe encore Yacine Kateb) ou Jean Sénac lui-même ${ }^{16}$.

\section{Terrasses}

Six ans séparent le dernier numéro de Forge de l'unique numéro publié de Terrasses. Durant cette période capitale marquée par l'étalement de la misère des campagnes algériennes, d'importantes avancées du nationalisme et l'apparition de nouvelles revues clairement désireuses «de nier catégoriquement la légitimité de cette répartition pratique des Algériens en deux groupes ${ }^{17} »$ les deux équipes fondatrices accompagnent de façon très différente la reconfiguration politique qui s'amorce : alors que Roblès et Safir se montrent proches de l'UDMA ${ }^{18}$, partisan d'une société multiculturelle et intégrative, Sénac adoptera progressivement, au contact de jeunes militants du MTLD ${ }^{19}$, une position plus radicale, favorable à une Algérie algérienne et indépendante.

Face à l'optimisme humaniste de Forge, aux ambitions pluriartistiques de Soleil, le titre Terrasses apparaît pourtant plus modeste, plus connotatif, évoquant un espace privilégié d'intimité et de passage, un lieu d'observation plus que d'action, une sorte de balcon sur la Méditerranée :

Nous avons longuement parlé avec Camus, lors de son séjour ici, de la nouvelle revue. Elle s'appellera Terrasses. Tu connais notre programme. Il y aura des textes de première grandeur, une sorte de «Journal à plusieurs voix " rédigé par nous tous, et d'importantes chroniques traitant des littératures méditerranéennes en mouvement (Afrique du Nord, Égypte, Palestine, Grèce, Liban, Espagne, Italie, Yougoslavie, etc.) et des littératures sud-américaines. [...] Nous avons, au cours de notre réunion du 21 décembre avec Camus, signé l'Éditorial de la Revue. [...] Veuxtu me dire si tu es d'accord sur ce texte ${ }^{20}$ ?

Ce texte introductif non signé affirme implicitement qu'il n'est plus temps pour des « inventaires passionnés ${ }^{21}$ » de ce qui a été réalisé et trop tard pour des visions utopiques de "libération des esprits » dans une destinée commune; que le rôle de l'intellectuel algérien ne peut être que de rester à l'affût, d'observer, en prenant un peu de hauteur, ce qui peut, ce qui va advenir. 
fig. 6 : Texte de présentation de Terrasses.

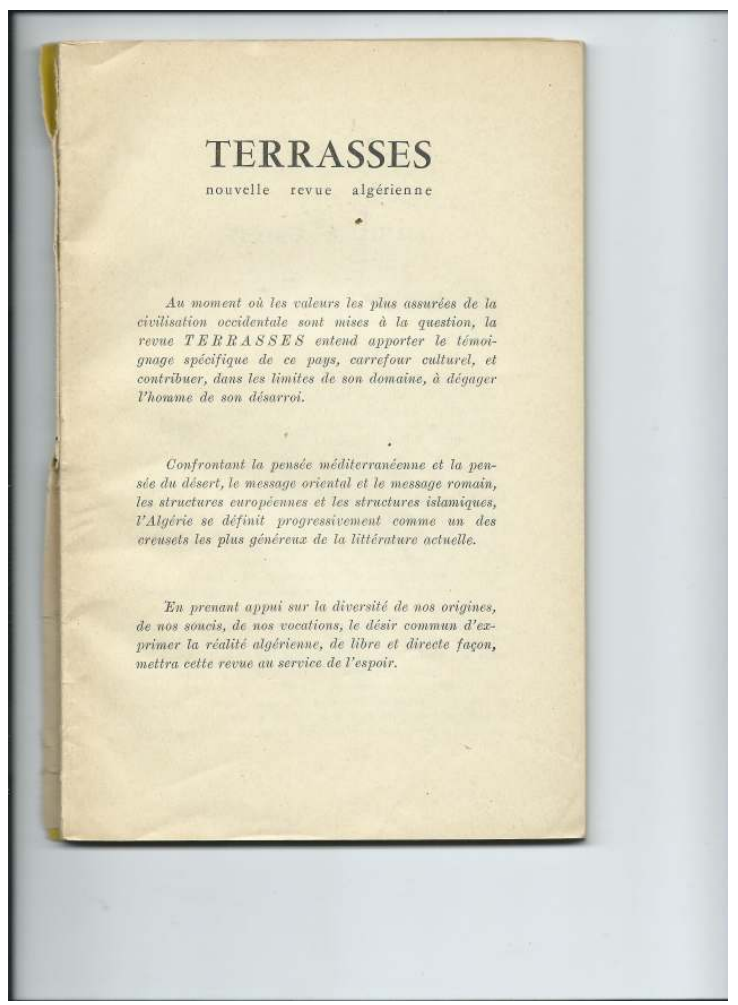

Fonds Patrimoine méditerranéen, BIU Montpellier, section Lettres.

Le texte que Sénac envoie à Mohammed Dib pour correction était plus long, plus emporté et plus lyrique : il y était question du respect et de la dignité que la littérature procure à l'homme, de « voix du perpétuel amour », de « dialogues douloureux », de « ménagements inutiles », etc.

On ne peut savoir la part prise par Camus dans cette rédaction; mais j'ai pu retrouver l'envoi fait à Mohammed Dib et la réponse de celui-ci, avec des annotations ${ }^{22}$ allant dans le sens d'une plus grande sobriété et d'un moindre lyrisme : 
fig. 7 : Brouillon du texte de présentation de Terrasses, annoté par M. Dib.

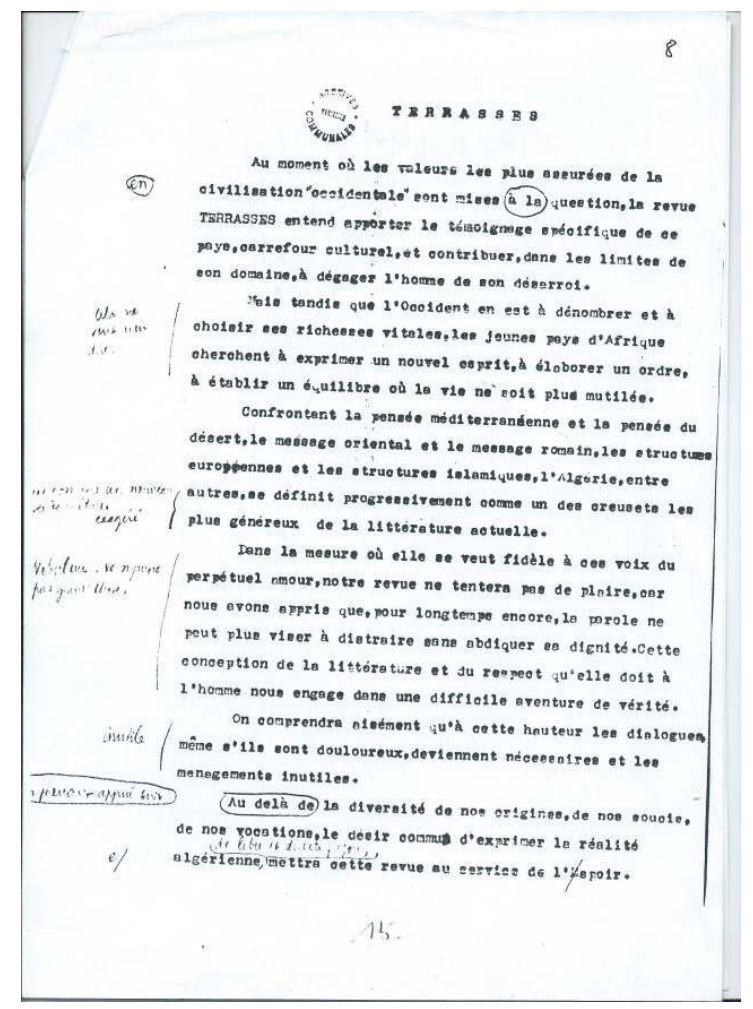

Fonds Jean Sénac, bibliothèque de l'Alcazar, Marseille.

Évolution du contexte social, mais aussi apport de la première revue : si sa durée de vie fut moindre que celle de Forge, le volume et la densité de Terrasses, son impact même, furent largement équivalents, dans un contexte prérévolutionnaire qui attire l'attention de tous sur ce qui se passe outre-Méditerranée: de passage à Paris, Dib note qu'« un intérêt croissant s'[y] manifeste chaque fois qu'il est question de l'Afrique du Nord. [...] Allant à la rencontre de cette curiosité, de cette inquiétude même, une revue algérienne pourrait recevoir un accueil très favorable qui ferait un effort de sincéritéén ${ }^{23}$.

En quelques années et grâce à des personnalités comme Emmanuel Roblès ou Gabriel Audisio, une jeune littérature maghrébine de langue française s'est révélée en métropole. Les débutants de Forge: Mouloud Feraoun, Mohammed Dib ou Kateb Yacine en Algérie, Ahmed Sefrioui au Maroc, Albert Memmi en Tunisie, sont devenus des partenaires réguliers et actifs de Terrasses, chacun de son côté s'efforçant en outre de mobiliser largement dans son pays. En métropole, où la revue est, selon Dib, jugée "brillante », le comité de rédaction prend contact avec Georges Bernanos, Gilbert Sigaux, Roland Barthes, Jean Servier, etc. À Memmi, sollicité pour un $n^{\circ} 2$ qui ne verra jamais le jour, Sénac demande :

Si vous connaissez de jeunes Tunisiens intéressants, n'hésitez pas à m'envoyer leurs textes. D'autre part, vous-même ne pourriez-vous pas (même sous pseudonyme) nous donner une chronique tunisienne (littéraire et même politique ${ }^{24}$ ). Qu'en pensez-vous ${ }^{25}$ ? 


\section{Une mort programmée}

Dans ce Landerneau culturel qu'est Alger, les relations interpersonnelles prennent une importance considérable, les engagements politiques séparent inévitablement et il convient de prendre garde à ce que l'on écrit. La moindre anicroche a des conséquences sans proportion avec les causes qui l'ont produite.

À Forge, ce sont les dissensions politiques déjà évoquées avec Julia et Alger républicain, privant la revue de l'appui financier et logistique capital de cette société, désireuse, selon Roblès, « de contrôler le contenu [de la revue] et de la politiser davantage, dans l'espoir d'élargir le public ", qui - associées au lâchage d'Hachette, tout puissant diffuseur du livre dans l'Afrique du Nord coloniale - conduiront à la disparition de la revue.

fig. $8 a$ et $8 b$ : Extraits des entretiens inédits d'E. Roblès avec $M$. Chavardès.

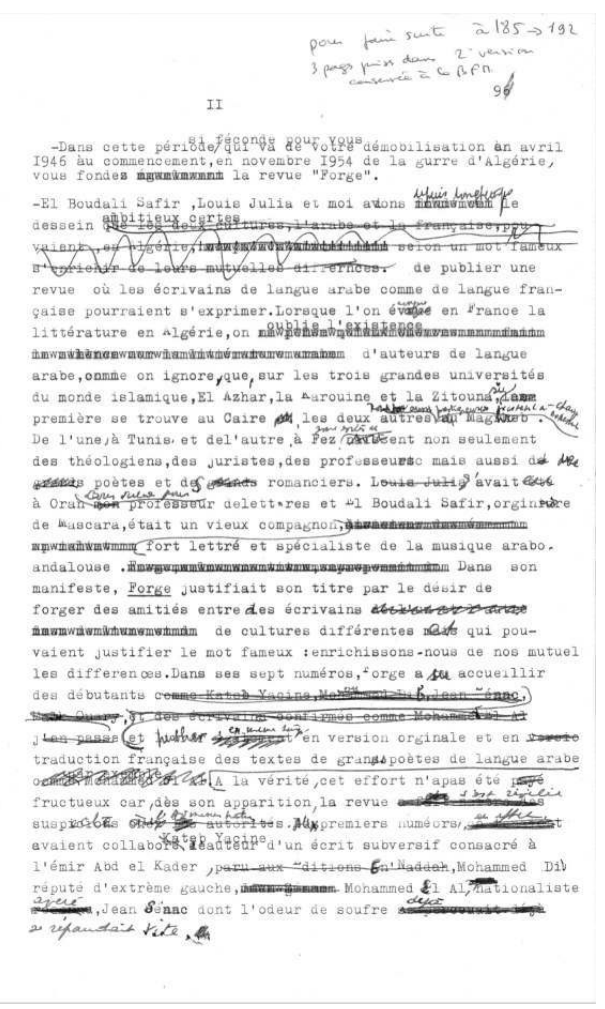




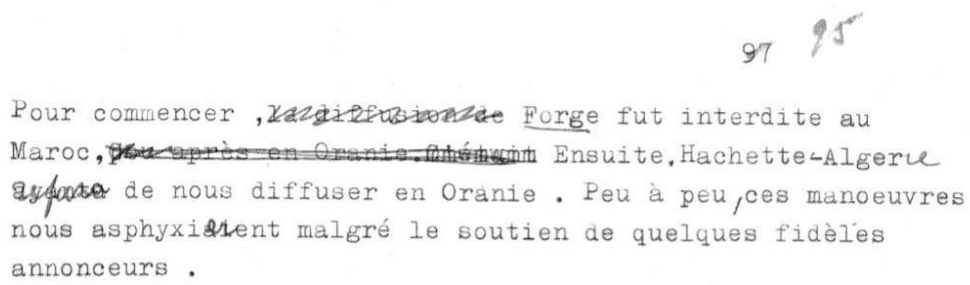

Bfm Limoges, fonds E. Roblès-Archives.

Il semblerait qu'il en ait été de même pour Terrasses, sur le plan politique du fait de la radicalisation progressive des opinions de Sénac qui vont le conduire à rompre avec $\mathrm{Camus}^{26}$, comme sur un plan strictement littéraire. Témoin ce différend avec Gabriel Audisio, écrivain reconnu et personnalité importante dans les milieux culturels de la colonie :

$\mathrm{Au} 2$, nous avions un texte d'Audisio sur les possibilités d'un théâtre algérien, mais ayant informé son auteur que notre critique était sévère pour sa pièce [La Clémence $d u$ Pacha] et que d'autre part son texte provoquerait des réactions au numéro suivant, Audisio s'est retiré. Tant pis - ou tant mieux. Notre revue se doit de parler clairement, franchement, hors tous les copinages et sans crainte de déplaire à quelques-uns. Sais-tu que La Clémence du Pacha est brandie comme l'étendard du théâtre algérien, à la grande colère de la majorité des Algériens. Terrasses, qui veut exprimer la réalité algérienne, devait prendre parti dans cette querelle, quitte à déplaire à ces messieurs du $\mathrm{G}$ [ouvernement] $\mathrm{G}$ [énéral]. Voilà qui est fait ${ }^{27}$.

Avec un texte de Sefrioui au titre significatif, une chronique de Jean Daniel sur la question nord-africaine et le « très beau texte de Feraoun » annoncé par Sénac à Dib ${ }^{28}$, le numéro 2 de Terrasses promet d'être plus engagé encore que le premier, de ne jamais éviter les confrontations, de susciter la polémique : 
fig. 9 : Projet de sommaire du $\mathrm{n}^{\circ} 2$, non paru, de Terrasses.

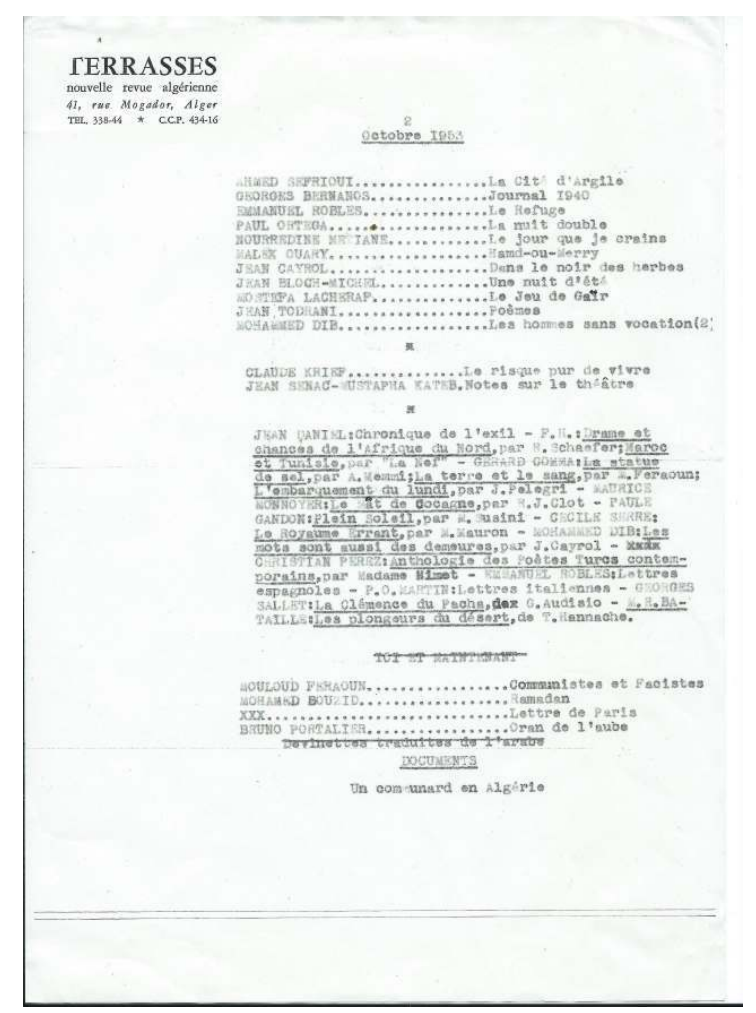

Arch. Albert Memmi, fonds privé.

Est-ce pour cette raison que ce volume ne vit jamais le jour? Ou bien à cause de « cette vaste opération menée par des "puissances occultes" pour empêcher la publication de tout livre jugé subversif venant des colonies » que Dib a cru découvrir au cours d'un voyage en France ${ }^{29}$ ? Il faut dire qu'entre 1947 et 1953, de leurs débuts dans Forge à leur collaboration à Terrasses, les Dib, Kateb, Sénac... ont mûri dans leurs convictions politiques, parfois divergentes, et sont devenus de plus en plus suspects pour l'autorité coloniale.

Toujours est-il qu'au début de l'hiver 1953-1954, un an avant qu'éclate la Révolution nationale, le second numéro est devenu pour à peu près tout le monde « une manière de mythe qui permet tous les espoirs, du moment qu'il n'existe pas $^{30}{ }^{30}$ !

Forge n'aura donc pas passé l'année et Terrasses n'aura vécu que l'espace d'un numéro mais l'échec ou la réussite d'une revue culturelle ne se mesurent-ils qu'à leur durée?

\section{BIBLIOGRAPHIE}

NACER-KHODJA, Hamid, Albert Camus - Jean Sénac, ou le fils rebelle, Paris, éd. Paris-Méditerranée, 2004. 
NACER-KHODJA, Hamid, Jean Sénac critique algérien, Alger, El Kalima, 2005.

RIVAS, Pierre, « La revue Forge (Alger, 1946-1947), un lien entre deux cultures », La Revue des revues , n 48 , automne 2012.

Collectif, Algérie, un rêve de fraternité, Paris, Omnibus, 1997.

\section{NOTES}

1. Voir le «Manifeste de l'Algérianisme », signé Jean Pomier, dans Afrique, ${ }^{\circ}$ 5, oct. 1924, p. 1-6 : «L'Algérianisme pourrait être, dans l'ensemble de la littérature d'empire, l'effort d'art local qui consisterait à étudier et à décrire les relations des diverses formes morales de l'Algérie. Ainsi défini, l'Algérianisme ne saurait être rabaissé par tout homme de bonne foi à de mesquines préoccupations d'école, ou comme on s'exprimerait ici de çofs littéraires. Il comporte, plus qu'une règle d'art, plus qu'un devoir littéraire, un devoir humain [...]. L'Algérianisme nous est, en effet, apparu [...] comme la féconde et anxieuse préparation de nos destins africains et, par là même, des destins de la Mère Patrie. [...] Il ne saurait donc s'agir pour un algérianisme pratique que de suivre assidûment l'évolution de nos milieux, de s'employer à faciliter leur décantation, à hâter la majorité mentale de l'Algérie, mais avec la préoccupation première que l'âme algérienne de demain, si elle doit avoir sa tonalité particulière, ne soit cependant qu'une composante de la clarté française. »

2. Dans une lettre inédite à Jean Sénac, Roblès parle d'Afrique comme d'« une confidentielle feuille de chou ".

3. Lancée par Gabriel Audisio et Albert Camus chez Charlot en décembre 1938, cette « revue de culture méditerranéenne » devait paraître tous les deux mois. Le $\mathrm{n}^{\circ} 1$, essentiellement poétique, associe des poètes fort connus de la Méditerranée occidentale (Garcia Lorca, Machado, Supervielle) et des proches de Camus et Charlot (Blanche Balain, Claude de Fréminville). La revue bénéficie d'un appui logistique et financier non négligeable de la jeune maison d'édition, ainsi que de placards publicitaires. Hélas, elle ne connaîtra qu'un seul numéro et sera ensuite victime de la guerre.

4. Forge, « À nos lecteurs ».

5. Y compris à l'encontre de collaborateurs de Forge, et quelques années plus tard des revues de Sénac, qu'il juge peu innovantes.

6. Même si, dans certaines correspondances ou certains entretiens, Roblès fait aussi état du lâchage de Hachette distribution et d'une interdiction de la revue au Maroc.

7. Projet éditorial de Soleil resté inédit, cité par Hamid Nacer Khodja, 2005.

8. Référence évidente à la prestigieuse Nouvelle Revue française.

9. «Vous n'avez pas oublié, j'espère, de demander de la publicité pour les romans nord-africains à Plon, au Seuil et à Gallimard. Sinon, faites-le tout de suite. » Lettre de Mohammed Dib à Jean Sénac, Tlemcen, 12 avril [1953].

10. Lettre de Sénac à Mohammed Dib, Alger, 9 avril 1953. Le poète vient d'hériter d'un petit pécule de 200000 francs.

11. Jean Sénac à Albert Memmi, lettre inédite, «Alger, $1^{\text {er }}$ octobre 1953 ». D’autres documents et correspondances attestent d'un projet de constitution d'une société de ce type avec Edmond Charlot, qui ne s'est pas consolé de l'échec de Rivages, qu'il tentera en vain de faire revivre une dizaine d'années plus tard.

12. S'il faut en croire le journal de Sénac, les deux écrivains, qui se connaissaient déjà, y ont discuté de l'utilité des revues et de l'expérience, en cours, de Forge. Par la suite, on les retrouvera l'un et l'autre au sommaire de leurs revues respectives, à côté d'artistes rencontrés à Sidi Madani. 13. « À nos lecteurs », in Forge. 
14. Sur ce type d'entreprises, désespérées en contexte colonial, lire notre introduction: " Plaidoyer pour quelques hommes de bonne volonté ", à l'anthologie : Algérie, un rêve de fraternité, Omnibus, Paris, 1997.

15. Les auteurs du texte de présentation de Terrasses s'approprieront cette formule, les «soucis [...] des uns et des autres » devenant « nos soucis ».

16. En réalité, la pénurie de plumes dans les deux langues est si grande que les directeurs de nos deux revues sont obligés d'écrire et de faire écrire sous pseudonymes pour donner l'illusion du nombre (voir ci-après lettre de Sénac à Albert Memmi)!

17. Manifeste de la revue Consciences algériennes, $n^{\circ} 1$, déc. 1950.

18. Union démocratique du manifeste algérien, parti modéré créé au printemps 1946 par Ferhat Abbas.

19. Mouvement pour le triomphe des libertés démocratiques, créé à l'automne 1946 par Messali Hadj.

20. Jean Sénac à Mohammed Dib, lettre inédite, « Alger 24 décembre 1952 ».

21. "À nos lecteurs", op.cit.

22. Lettre du 7 mars 53 : "Voici avec quelques annotations le projet d'édito que tu me soumets », accompagnée du projet du texte de présentation annoté (fig. 7).

23. Lettre inédite de Mohammed Dib à Jean Sénac, « Tlemcen, 26 décembre 52 ».

24. En pleine période d'agitation au Maroc et en Tunisie, Sénac cherche à politiser davantage sa revue : « Nous ne traiterons pas de la question marocaine et nous contenterons de publier un très beau texte d'Ahmed Sefrioui. J'en souffre " (lettre inédite à Mohammed Dib, « Alger le $1^{\mathrm{er}}$ octobre $1953 »)$. Cet engagement contribua sans doute à éloigner de Terrasses certains contributeurs potentiels.

25. Lettre citée du $1^{\mathrm{er}}$ octobre 1953. De son côté, Mohammed Dib rassure Sénac: «Bien sûr, je demanderai des textes autour de moi, surtout de jeunes gens inconnus» (lettre inédite, « Tlemcen le 3 janv. 53 »).

26. Voir à ce sujet Albert Camus - Jean Sénac, ou le fils rebelle d'Hamid Nacer-Khodja, Paris, éd. ParisMéditerranée, 2004.

27. Jean Sénac à Mohammed Dib, lettre inédite, «Alger, le 6 juillet 1953 ».

28. Dib, lui-même communiste, s'alarme, du reste, à la lecture de son seul titre : «Le sommaire du $n^{\circ} 2$ est splendide ! [...] Bravo et bon courage ! Cependant la présence d'un texte annoncé, celui de Feraoun ("Communistes et fascistes") a piqué ma curiosité. Il faudrait faire attention à ce que tu publieras là, qui pourrait être lourd de conséquences. Une franchise amicale m'autorise à te dire qu'un tel titre est déjà inacceptable. Prends bien garde à la voie où la revue s'engagera de la sorte » (lettre inédite à Jean Sénac, « Tlemcen, le 7 oct. 53 »).

29. Lettre inédite à Jean Sénac, « Tlemcen, le 17 oct. 53 ».

30. Lettre inédite de Dib à Jean Sénac, « Tlemcen, le 9 nov. 53 ».

\section{RÉSUMÉS}

Forge et Terrasses, publiées à quelques années près dans le même contexte de l'Algérie coloniale, sont deux revues assez significatives des difficultés que peut rencontrer une entreprise d'écriture collective aux prises avec les échecs politiques et l'aporie culturelle.

L'objet de cet article est de cerner de manière comparative les conditions de gestation des deux 
revues, considérées comme des processus de création collective, de mettre en évidence les moyens de leur fonctionnement, leurs ambitions et leurs engagements, et de tenter de mesurer à travers leurs archives et les éléments de leur genèse la part de réussite et les raisons de leur échec relatif.

INDEX

Mots-clés : revues littéraires, création collective, culture et colonie, engagement, Algérie coloniale, Mohammed Dib, Jean Sénac, Edmond Charlot, Emmanuel Roblès

\section{AUTEUR}

\section{GUY DUGAS}

Chercheur associé à l'équipe Manuscrits francophones, ITEM (CNRS-ENS) 